\title{
LEARNBOTICS: UMA NOVA ABORDAGEM DE ENSINO PARA ROBÓTICA
}

Leandro S. O. Nozela1; Frederico Garcia de Oliveira²; Bruno de Souza²; Marco Antonio dos Reis'.

${ }^{1}$ Centro Universitário SENAI-CIMATEC; Salvador/BA; nozela.leo@gmail.com

${ }^{2}$ Centro Universitário SENAI-CIMATEC; Salvador/BA

Resumo: Este artigo tratará de uma nova abordagem de ensino, voltada para a robótica, aliando aprendizado prático e teórico ao mesmo tempo que aborda conceitos básicos da robótica aplicada. A abordagem apresentada será baseada em uma junção de metodologias de ensino focadas no aprendizado prático como exemplo o movimento maker, o PBL e o TBL. O conteúdo teórico foi escrito em linguagem acessível, e disposto em formato de tutoriais e apostilas disponíveis em domínio virtual. O kit físico será dividido em módulos complementares de montagem, tendo como principal diferencial a união da prática com a teoria de forma gradual. $O$ kit de aprendizado promete ser um bom precursor de avanços no ensino da robótica.

Palavras-Chave: Robótica; Ensino; Teoria; Prática.

\section{LEARNBOTICS: A NEW APPROACH ON TEACHING ROBOTICS}

\begin{abstract}
This paper aims to describe a new approach on teaching robotics, which combines theory and hands-on learning, while addressing basic robotics concepts. This approach will be based on a junction of methodologies which focus on theoretical teaching in addition with methodologies which focus on practical learning. The theoretical content was written in a simplified language and was displayed in a tutorial format and made available in an online environment. The physical kit will be divided in complementary and gradual assembling steps that will result in a differential robot. This new approach focuses on simplifying specific robotics contents while combining theoretical and practical learning, which may serve as a reference for future enhancements into teaching robotics.
\end{abstract}

Keywords: Robotics; Teaching; Theory; Practical. 


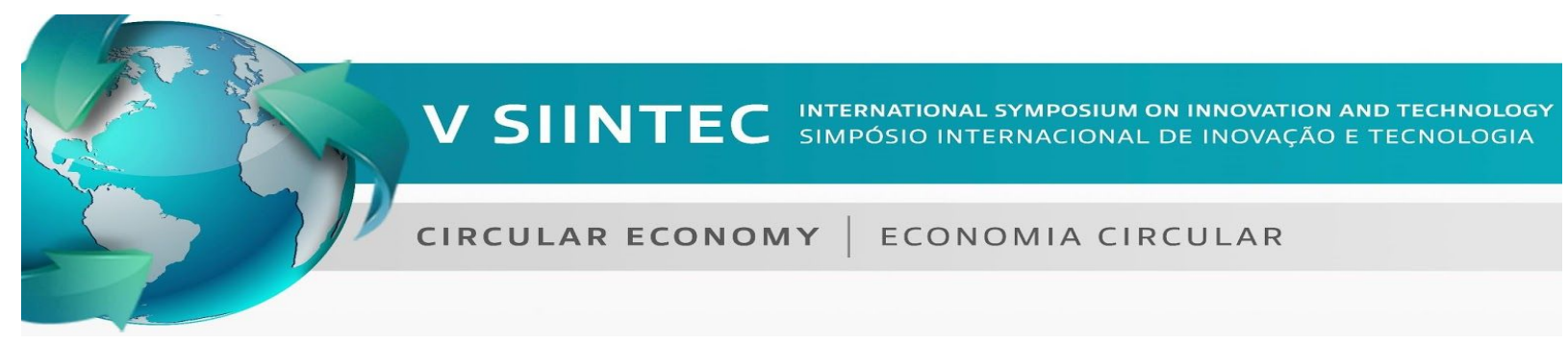

\section{INTRODUÇÃO}

Desde 2010 há um crescimento exponencial da utilização de sistemas robóticos na indústria, alcançando cifras de US\$ 16.2 bilhões no mercado global. Hoje a densidade de robôs, na indústria automotiva por exemplo, demonstra a crescente utilização destes sistemas nas áreas produtivas. No Japão, para cada 10000 empregados há 1158 unidades robóticas. Quando se expande para uma comparação global da indústria de maneira geral, este número cai para 225 robôs por cada 10000 empregados, porém um número que apresenta um crescimento acentuado. ${ }^{[1]}$

Uma outra área na qual se evidencia um crescimento do uso de sistemas robóticos é a de petróleo e gás, que vem apresentando altos investimentos em aplicações envolvendo a utilização de robôs. Algumas possíveis explicações para isso permeiam o crescimento da demanda, e possível escassez, de recursos derivados de combustíveis fósseis. Diante disso, a indústria de petróleo e gás tem buscado soluções robóticas para aumentar a sua produtividade e mitigar alguns riscos envolvidos nos processos de extração. ${ }^{[2]}$

Atualmente, a maioria das tecnologias que vêm sendo desenvolvidas focam em inspeção e manutenção. Essas operações vinham sendo primariamente baseadas em ações via teleoperação, onde um operador controla o robô à distância. Todavia, esse cenário tem se modificado, e um exemplo atual de um robô para a indústria de petróleo e gás é o FlatFish, que tem como principal aplicação a inspeção visual externa de tubulações em águas de até $300 \mathrm{~m}$ de profundidade de forma autônoma, ou seja, que dispensa o controle de um operador a distância. Esse tipo de robô é conhecido como um AUV (do inglês: Autonomous Underwater Vehicle).

Do ponto de vista econômico, segundo o resumo executivo World Robotics 2018 da International Federation of Robotics de 2018, houve uma crescente utilização de sistemas robóticos e autônomos na nossa sociedade. A demanda global de robôs tem crescido severamente, com estimativa de acréscimo de $14 \%$ ao ano até 2021. [1]

Esse aumento na demanda está presente em todas as áreas da indústria, porém com maior relevâncias nos setores eletroeletrônico e automotivo, de onde vêm as maiores expectativas de inovação por parte da população. Este fato provoca uma reflexão de que, talvez, a automação e a utilização de robôs tenderão a ocasionar o desemprego estrutural. Porém em contrapartida há uma crescente necessidade de profissionais para áreas que envolvem a robótica, desde a visão computacional, programadores, simulação computacional, frameworks de robótica, sistemas eletrônicos embarcados, engenharia mecânica, entre outros. A partir disso, 
- mercado tende a se adequar a estas necessidades, e consequentemente, profissionais de diversas áreas tendem a buscar especializações em robótica.

Há ainda uma notória dificuldade em capacitar trabalhadores para lidar com tecnologias com as quais não tiveram contato durante o ensino médio e fundamental, e mesmo quando tiveram, provavelmente, apresentou-se de uma forma limitada. Um dos motivos para tal, é que a atual abordagem de ensino não se preocupa, por exemplo, com a desmistificação de alguns conceitos atuais, como por exemplo: Sistemas Autônomos, Big Data e Machine Learning, que mesmo não abordados em sala de aula, os alunos acabam tendo contato através de pesquisas em meios digitais durante seus estudos.

Foi através desse cenário que nasceram os movimentos Maker e STEM, oriundos da metodologia Do It Yourself (em português: faça você mesmo). Ambos valorizam a possibilidade de utilizar das informações obtidas por pesquisa e conteúdo online de fácil acesso para fazer projetos com as próprias mãos, seja com ajuda de um computador, impressora 3D ou ferramentas, visando aumentar a atratividade para as áreas da ciência e desmistificar tabus relacionados à dificuldade de se aprender novas tecnologias. Como resultado, mais jovens e adultos interessam-se por tecnologia, seguem carreiras na área e aumentam o número de profissionais qualificados no mercado.

Ainda no campo da pedagogia, o ensino da robótica é interdependente de aulas no formato da pedagogia clássica, porém melhor aproveitado quando associado a atividades práticas em grupo. Por este motivo considera-se que o movimento Maker e algumas metodologias de ensino de robótica são baseadas na concepção de Lev Vygotsky, onde o sujeito é considerado um ser não só ativo como também interativo, porque adquire conhecimentos a partir de relações intra e interpessoais, exercitando o que o homem tem de melhor: a criatividade. Segundo a concepção de Vygotsky, a aquisição de conhecimentos se dá pela interação do sujeito com o meio. ${ }^{[3-4]}$

Essa associação visa apresentar de forma menos abstrata conceitos abordados nas aulas teóricas e propor compartilhamento de aprendizagem e fomentar o trabalho em grupo entre os alunos dos níveis Fundamental Il e Médio.

Percebendo essa nova dinâmica da educação, e a visível dificuldade em encontrar informações simplificadas sobre robótica, este artigo terá como objetivo propor uma nova abordagem de ensino, voltada para a robótica, nos níveis Fundamental II e Médio, contendo tutoriais, desafios e kit didático de aprendizagem prática, que envolve conceitos básicos da robótica aplicada, utiliza o framework ROS e aplica conceitos de cinemática e visão computacional. 


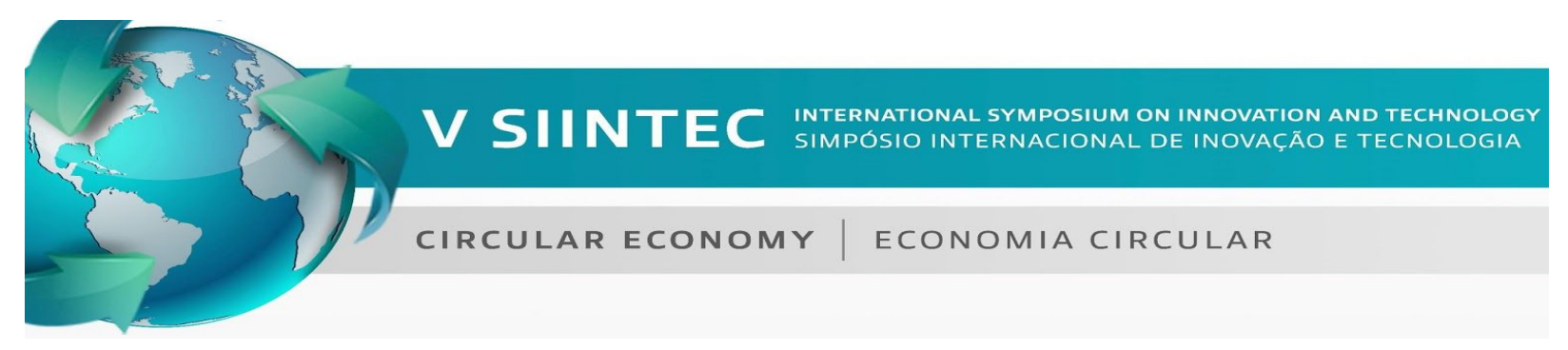

\section{METODOLOGIA}

Fora realizado um levantamento de referências que tratam das metodologias pedagógicas e seu histórico, abordando desde as pedagogias iniciais como a Tradicional e a Renovada, até metodologias de ensino mais atualmente aplicadas no ensino de robótica, como o PBL (do inglês Project-based Learning) e a TBL (do inglês Team-based Learning). ${ }^{[5-7]}$

A partir deste estudo, a metodologia apresentada pelo projeto Learnbotics foi proveniente de uma correlação entre as metodologias estudadas com o intuito de propor uma nova abordagem ao problema em questão, que perpassa por entre os métodos mais atuais de ensino aplicado à robótica, porém não ignorando a grande contribuição que métodos mais clássicos podem vir a apresentar.

A abordagem apresentada será baseada em uma junção de conceitos desenvolvidos por metodologias de ensino mais voltadas para o aprendizado prático, principalmente o movimento maker atrelados a concepções de Vigotsky de propor ao aluno atividades práticas a fim de construir o conhecimento correlacionando atividades práticas e teóricas. Pode-se citar também PBL, associada a metodologias mais clássicas e teóricas, como por exemplo a pedagogia Tecnicista, e usará ainda proposição de ações baseadas em métodos considerados informais como o DIY (do inglês Do It Yourself) e fora influenciada diretamente pelo proposto pelos precursores do movimento STEM (do inglês Science, Technology, Engineering and Mathematics). ${ }^{[5,8,9]}$

Figura 01 - Representação da metodologia.

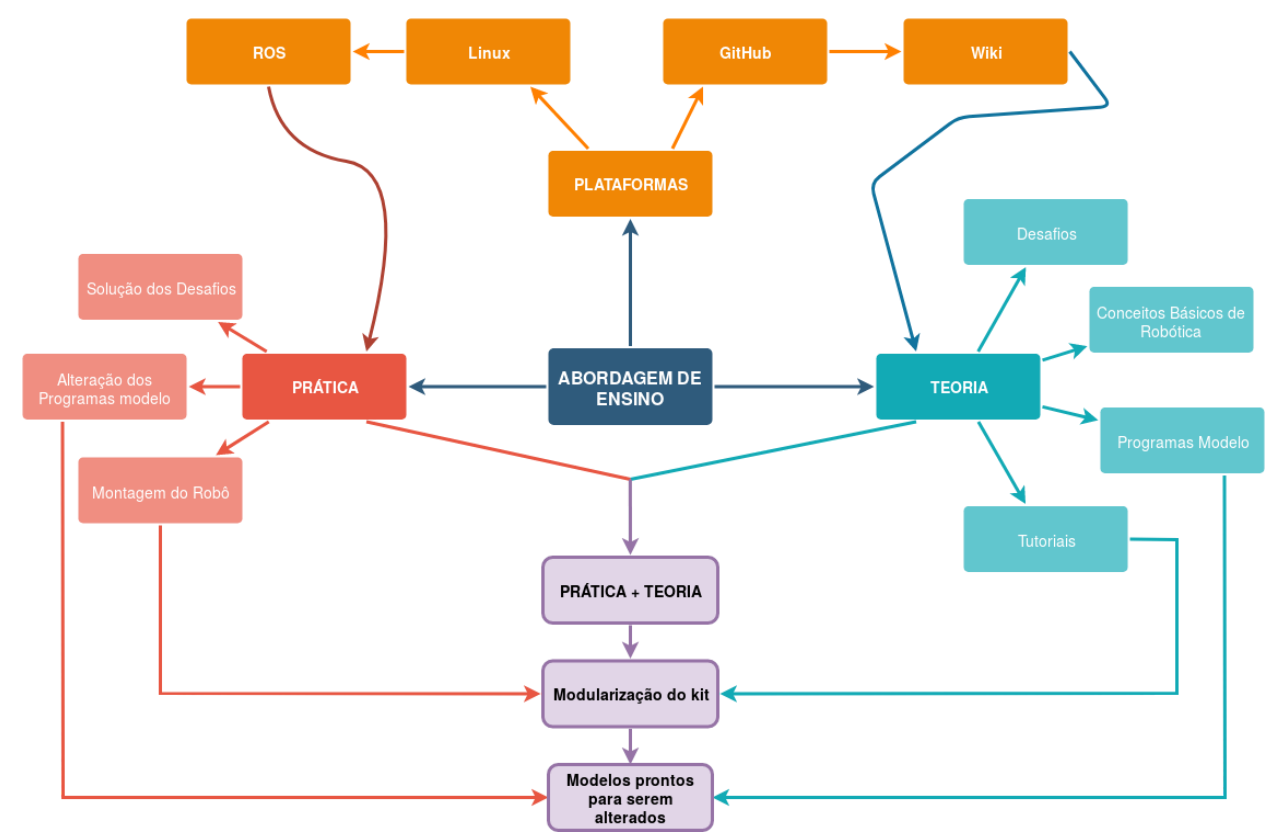

Fonte: Autores, 2019. 
A proposta de desenvolvimento será baseada na figura 01 acima, que apresenta os macro itens envolvidos, assim como uma exemplificação de como essas informações serão apresentadas ao estudante. Estes macro itens são subdivididos da seguinte maneira: Na parte teórica serão apresentados ao estudante conceitos básicos de robótica, baseados nas principais bibliografias e documentos que são normalmente utilizados pela comunidade de robótica, e já na parte prática, o aluno contará com um kit físico para ajudar a absorção dos conteúdos apresentados.

Do ponto de vista teórico, um exemplo do que será disponibilizado são os tutoriais do Framework ROS (do inglês Robot Operating System), que são elaborados pela própria comunidade do ROS. Todavia, não existem versões escritas em português, ou em uma linguagem que se preocupe em ser simplificada, o que dificulta o acesso à essas informações. Assim, a proposta é de utilizar estes tutoriais como inspiração e referência de conteúdo para produção de material escrito em linguagem mais acessível e em português.

Além da produção de tutoriais, há ainda a proposta de apresentar scripts modelo, escritos em python, que servirão como base para explicações de conceitos de programação. O foco destes, será de mostrar aos alunos como utilizar métodos e funções próprias do framework. Estes scripts estarão envolvidos diretamente com outro macro item, que é o aprendizado prático. A parte prática tem como foco o estudo e a alteração dos scripts, proporcionando assim o desenvolvimento do estudante em programação.

Cada script modelo estará atrelado a um desafio. Para que o estudante solucione estes desafios, a proposta é que ele leia, interprete e altere o programa para realizar uma tarefa específica que vai um pouco além da apresentada pelo script original.

Visando dar forma e visual à conceitos muitas vezes abstratos, o que é interessante na abordagem, é que, atrelado a essas alterações haverão dispositivos reais para demonstrar se houve ou não êxito. Alguns exemplos de dispositivos com os quais os estudantes poderão interagir são servomotores e uma câmera, o que facilitará uma absorção dos conceitos apresentados.

Para alcançar estes objetivos de aliar as macro áreas, o estudante terá contato também com uma plataforma online, o GitHub, onde encontrará todos os tutoriais e materiais complementares desenvolvidos. Paralelo à isso, na parte física, o estudante também terá um kit físico para montagem gradual de um robô com movimentação diferencial. Esta montagem será modular e será regida pelo seu andamento nos tutoriais. O quadro 01 apresenta os componentes presentes no kit. 
\begin{tabular}{l|l} 
CIRCULAR ECONOMY & ECONOMIA CIRCULAR
\end{tabular}

Quadro 01 - Relação dos Componentes presentes no Kit Físico.

\begin{tabular}{|c|c|c|c|c|c|c|c|}
\hline Componentes & $\begin{array}{c}\text { Raspiberry } \\
\text { Pi3B }\end{array}$ & $\begin{array}{c}\text { Dynamixel } \\
\text { MX-28 }\end{array}$ & Câmera RGB & $\begin{array}{c}\text { Rodas } \\
\text { emborrachadas }\end{array}$ & $\begin{array}{c}\text { Roda de } \\
\text { apoio }\end{array}$ & $\begin{array}{c}\text { Cabos e } \\
\text { conexões }\end{array}$ & Bateria \\
\hline Quantidade & 1 & 2 & 1 & 2 & 1 & $\mathrm{x}$ & 1 \\
\hline
\end{tabular}

Fonte: Autores, 2019.

A escolha da Raspberry Pi 3.0B se deve por conta da alta disponibilidade no mercado, por apresentar um custo baixo se comparado com outros computadores que serviriam para a mesma finalidade, e principalmente por apresentar poder computacional suficiente para o funcionamento do ROS e das bibliotecas de processamento de imagens que serão utilizadas.

Na Raspberry Pi será utilizado o sistema operacional Raspbian por ser o mais otimizado para a plataforma, por ser derivado de um sistema Linux, e por funcionar aliado ao ROS Kinetic, que é hoje a distribuição do ROS mais utilizada pela comunidade da robótica.

O estudante irá receber a Raspberry com todas as bibliotecas necessárias para funcionamento e alteração dos scripts já instaladas, assim como o framework também já instalado, poupando assim o desestímulo inicial que tende a ocorrer no primeiro contato com estas ferramentas.

Serão apresentados os servomotores Dynamixel, por serem amplamente utilizados em projetos robóticos de inovação. Isso ocorre por conta da maior facilidade de integrar estes servos com o ROS, sem precisar utilizar ferramentas mais complexas, como desenvolvimento de drivers e controle PWM (do inglês, Pulse Width Modulation). Em um dos scripts modelo, por exemplo, irá ser apresentada a forma de se comunicar com os servos, juntamente à introdução da cinemática de robôs diferenciais.

A câmera RGB será utilizada para facilitar a introdução de conceitos de visão computacional e suas ferramentas, como a biblioteca do OpenCV (do inglês, Open Computer Vision). Haverão scripts modelo apresentando a integração entre ROS e OpenCV, além da proposição de desafios utilizando conceitos básicos de visão computacional. Ao final deste módulo, será apresentado para o estudante o desafio final do kit, que se tratará de uma integração dos conhecimentos apresentados previamente. 


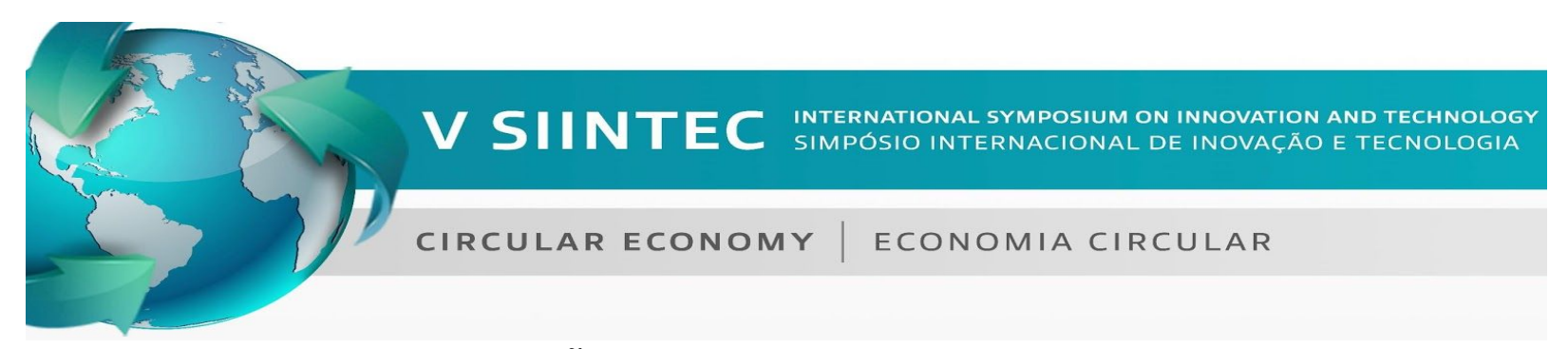

\section{RESULTADOS E DISCUSSÃO}

O conteúdo teórico foi escrito em linguagem acessível, e disposto em formato de tutoriais e apostilas disponíveis em domínio virtual. $\mathrm{O}$ foco destes tutoriais é apresentar conceitos de uma forma simples, direta e utilizando uma linguagem descomplicada. Ademais, todos os tutoriais estarão disponíveis no Github, em formato de wiki, em repositório aberto, escritos em português, de forma a promover a acessibilidade do conteúdo à comunidades lusófonas em geral.

O kit físico foi dividido em módulos complementares de montagem, resultando em um robô com movimentação diferencial. Por se tratar de um robô simples, que foi pensado para ser fabricado através de manufatura aditiva, apresenta fácil montagem englobando todos os componentes do kit físico e tangenciando conceitos de poka yoke, a fim de propiciar ao usuário um aprendizado mais amigável. Este kit se torna um diferencial quando estimula o aluno a buscar uma maior interação com a robótica ao passo em que exercita a sua criatividade através de objetos físicos que interagem com conceitos abstratos.

Esta modularização será atrelada à progressão do aluno. Na primeira parte ele terá acesso, principalmente ao computador, aprendendo seu funcionamento básico. Na segunda parte terá acesso aos servomotores e aprenderá a conectá-los ao computador e a enviar comandos a partir de scripts. O próximo passo será adicionar a webcam e aprender a utilizar ferramentas de visão computacional. $O$ estudante continuará tendo acesso a módulos complementares passo a passo a medida que vai avançando nos conceitos, até que chegue ao desafio final que irá integrar todos os passos apresentados anteriormente.

\section{CONCLUSÃO}

Ao combinar metodologias de ensino diferentes, focando principalmente na parte prática do aprendizado e visando dar forma e visualização a conceitos muitas vezes estritamente abstratos, essa nova abordagem de aprendizado apresenta características apelativas à um público mais jovem. Algumas dessas características que podem ser identificadas são a utilização de uma linguagem mais acessível, e um estímulo à criatividade e ao desenvolvimento de habilidades práticas.

Ao aplicar ferramentas que são utilizadas por profissionais da área, como por exemplo um sistema operacional baseado em Linux, o framework ROS e plataforma de versionamento como o Github, o estudante terá desde cedo contato com conceitos e habilidades requisitadas pelas funções desempenhadas por um profissional da área de robótica. 
Há ainda bastante trabalho a ser realizado do ponto de vista da democratização dos conhecimentos abordados, de forma a dispô-los de uma maneira ainda mais clara e simplificada, porém, conclui-se que essa nova abordagem promete ser uma boa precursora de avanços na democratização do ensino da robótica à estudantes pré-graduados.

\section{REFERÊNCIAS}

[1] INTERNATIONAL FEDERATION OF ROBOTICS. Executive Summary World Robotics 2018 Industrial Robots. [S. I.], 2018. Disponível em: https://ifr.org/downloads/press2018/Executive_Summary_WR_2018_Industrial_Robo ts.pdf. Acesso em: 15 maio 2019.

[2] SHUKLA, Amit; KARKI, Hamad. Application of robotics in onshore oil and gas industry-A review Part I. Robotics and Autonomous Systems, v. 75, p. 490-507, 2016.

[3] MATARIC, Maja J. Robotics Education for All Ages. 2004. Disponível em: <https://www.aaai.org/Papers/Symposia/Spring/2004/SS-04-01/SS04-01-004.pdf>. Acesso em: 20 jul. 2019.

[4] PALANGANA, Isilda Campaner. Desenvolvimento e Aprendizagem em Piaget e Vigotski: A relevância Social. 6. ed. São Paulo, SP: Summus Editorial. 2015.

[5] LARCHERT, Jeanes Martins. Pedagogia: Didática e Tecnologia I. Módulo 2, volume 5. Ilhéus - BA: UAB/UESC, 2010. 80p.

[6] KARAHOCA, D. et al. Robotics teaching in primary school education by project based learning for supporting science and technology courses. Elsevier Ltd., 2010. Disponível

em: <https://www.sciencedirect.com/science/article/pii/S1877050911000263>. Acesso: 10 ago 2019.

[7] DILlENBOURG, P. et al. Collaborative Learning: Cognitive and Computational Approaches, Advances in Learning and Instruction Series. New York, NY: Elsevier Science, Inc. 1999.

[8] Wolf \& McQuitty. Understanding the Do-It-Yourself Consumer: DIY Motivation and Outcomes. Academy of Marketing Science Review, 2011.

[9] PUGLIESE, Gustavo. STEM: O movimento, as críticas e o que está em jogo. Porvir, $2018 . \quad$ Disponível em: <http://porvir.org/stem-o-movimento-as-criticas-e-o-que-esta-em-jogo>. Acesso: 09 ago 2019. 